\title{
Robust Facial Alignment for Face Recognition
}

\author{
Kuan-Pen Chou ${ }^{1}$, Dong-Lin $\mathrm{Li}^{1}$, Mukesh Prasad ${ }^{2}$, Mahardhika Pratama ${ }^{3}$, \\ Sheng-Yao Su${ }^{1}$, Haiyan $\mathrm{Lu}^{2}$, Chin-Teng Lin ${ }^{2}$, and Wen-Chieh Lin ${ }^{1}$ \\ ${ }^{1}$ Department of Computer Science, \\ National Chiao Tung University, Taiwan \\ ${ }^{2}$ Faculty of Engineering and Information Technology, \\ University of Technology Sydney, Sydney, Australia \\ ${ }^{3}$ School of Computer Science and Engineering, \\ Nanyang Technological University \\ \{kpchou.cs00g@g2.nctu.edu.tw, lazybones@g2.nctu.edu.tw, \\ mukesh.prasad@uts .edu.au, mpratama@ntu.edu.sg, mikea1d2c3@gmail.com, \\ haiyan.lu@uts.edu.au, chin-teng.lin@uts.edu.au, wcline@cs.nctu.edu.tw\}
}

\begin{abstract}
This paper proposes a robust real-time face recognition system that utilizes regression tree based method to locate the facial feature points. The proposed system finds the face region which is suitable to perform the recognition task by geometrically analyses of the facial expression of the target face image. In real-world facial recognition systems, the face is often cropped based on the face detection techniques. The misalignment is inevitably occurred due to facial pose, noise, occlusion, and so on. However misalignment affects the recognition rate due to sensitive nature of the face classifier. The performance of the proposed approach is evaluated with four benchmark databases. The experiment results show the robustness of the proposed approach with significant improvement in the facial recognition system on the various size and resolution of given face images.
\end{abstract}

Keywords: face recognition, face alignment, facial feature localization and sparse representation classifier

\section{Introduction}

Face detection is a challenging and important issue to the facial recognition system In the real world application for security and safety purposes. The accurate detection of the face image increases the performance of the facial recognition system. Viola and Jones [1] introduced a fast object detection method based on a boosted cascade of haar-like features. This technique is widely used in the area of face recognition and detection research due to its computational capabilities and considerable performance. However, the variations of human faces based on different environment and conditions are still a challenging task in the real-world facial recognition system. The changes of facial expression, and pose, which are caused by uncontrolled environments deteriorate the accuracy of facial recognition system. Numerous approaches have been developed to handle the following 
face variation: (1) appearance-based methods [2]-[4], (2) train multiple detectors for each poses of human face [5]-[8], (3) nonlinear regression method [9]-[14], (4) manifold embedding method [12]-[17], and (5) geometrical analysis [18][19]. This paper uses a very simple geometric information to evaluate the human facial pose. For geometric approach, the accuracy of face feature point is very important. To find an accurate location searching for the face feature point and achieving a computational compatibility, an ensemble of regression tree based on gradient boosting technique [20] is applied in the proposed approach . The proposed approach precisely finds the face feature point of the test image and perform the facial correction by a geometrical transform technique.

The reminder of the proposed approach is organized as follows: Section 2 provides detail descriptions of the proposed approach which include architecture of the proposed approach, face alignment system, and face correction algorithm. Section 3 shows the experimental results on various facial benchmark databases.and finally the conclusions are covered in Section 4.

\section{Proposed System}

\subsection{Face Alignment System}

The architecture of the proposed system is shown in Fig. 1. At first, the proposed system localizes the landmark of input face obtained from the face detector [1] via a regression tree based method. According to the position of landmarks, the facial orientation can be easily estimated from two-dimensional geometric analysis. As the face orientation is determined, the input face is normalized to the frontal pose by compensating the face misalignment on each axis.

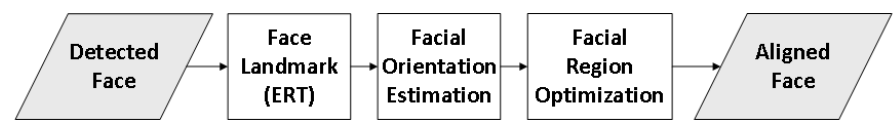

Fig. 1. The flowchart of our face correction system

\subsection{Face correction algorithm}

Compensation of pitch: The rotation motion of face can be represented as pitch (rotation about the $x$-axis), yaw (rotation about the $y$-axis), and roll (rotation about the $z$-axis). In order to explicitly explain the proposed approach, we suppose that the $z$-axis is the frontal orientation of human face; that is, the frontal pose can be described in $x-y$ plane, as shown in Fig. 2.

Fig 3 shows a geometric perspective that represents the projection from $3-\mathrm{D}$ human face onto 2-D image plane. Let $f$ is the focus distance of camera, $\gamma$ is the angle of pitch, $N$ is tip of the nose, $N B$ is the location of nose bridge, $U L$ is the 


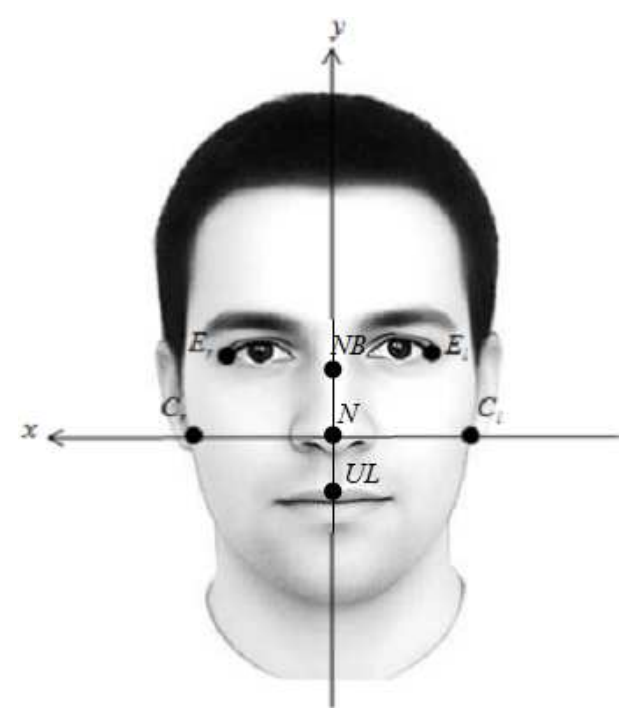

Fig. 2. The position of facial feature points

central point of upper lip, and $n, n b$, and $u l$ are the projection of each points in 2-D image plane. In $\gamma=0^{\circ}$ case, $U L$ should locate on the $z=0$ plane if we set $N B$ as the origin,

For the convenience of computation, we define two parameters as following:

$$
\begin{aligned}
& y_{u}=n b-n \\
& y_{d}=n-u l
\end{aligned}
$$

where $y_{u}$ is the nose length, $y_{d}$ is the distance of nose and upper lip in 2-D image plane.

Next, observe the influence of pitch angle $\gamma$ on the facial feature in 2-D image plane. Let the distance of $N B$ and $U L$ is facial feature length, and now define the ratio of nose length and facial feature length $\hat{P}$ as follows:

$$
\hat{P}=\frac{y_{u}}{y_{u}+y_{d}}
$$

According to the experimental results, the proposed system only aligns the face images in the case of $\gamma>0^{\circ}$ that the facial feature length is shortened by the effect of pitch rotation. The compensation formula is shown below:

$$
\begin{gathered}
\text { Center }_{\text {pitch }}\left(x, y^{\prime}\right)=\text { Center }_{\text {origin }}(x, y+\Delta y) \\
\text { Height }_{\text {pitch }}\left(x, y^{\prime}\right)=\text { Height }_{\text {origin }}-\Delta y \\
\Delta y= \begin{cases}y_{u}, & \text { if } \gamma>0^{\circ} \\
0, & \text { if } \gamma \leq 0^{\circ}\end{cases}
\end{gathered}
$$




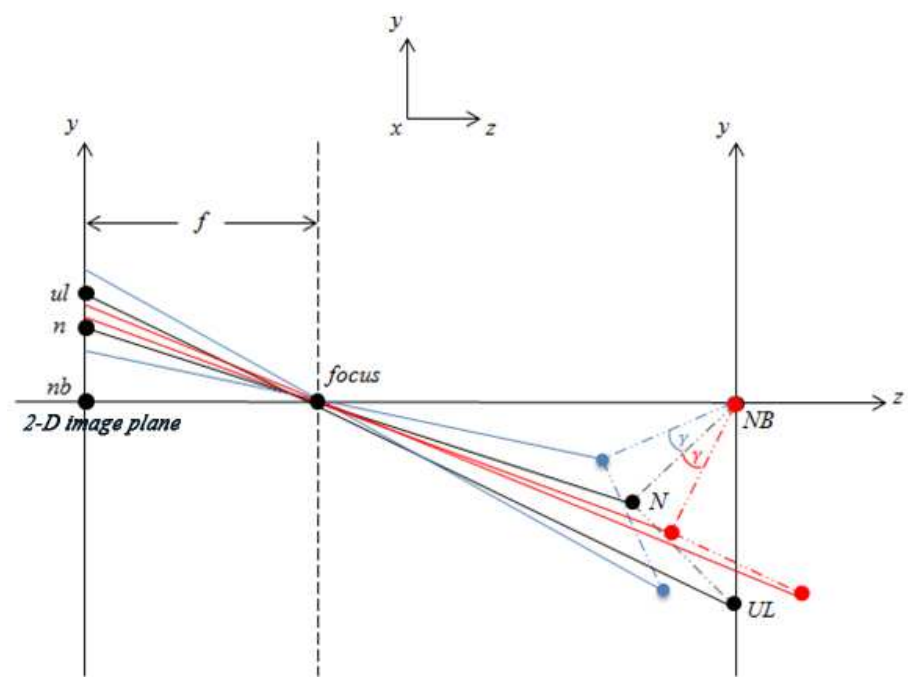

Fig. 3. The position of facial feature points

Compensation of yaw: Similarly as the case of pitch angle, the frontal human face is described in $x-y$ plane. In Fig. $2, C_{r}$ and $C_{l}$ are left and right side point of the face, respectively. $E_{r}$ and $E_{l}$ are outer corner of right and left eyes, respectively. In Fig. $4, \beta$ is the angle of yaw, $c_{r}, c_{l}, e_{r}$, and $e_{l}$ are the projection of each points in 2-D image plane.

According to the geometric perspective for Fig. 4, the projection terms $c_{r}$ and $c_{l}$ can be described as:

$$
\begin{gathered}
c_{r}=\frac{f X_{c}}{Z-Z_{c}}=\frac{f{C_{r}}^{-} E_{r} \cos \beta}{Z-Z_{c}} \\
c_{l}=\frac{f X_{c}}{Z+Z_{c}}=\frac{f C_{l}^{-} E_{l} \cos \beta}{Z+Z_{c}}
\end{gathered}
$$

and the yaw angle $\beta$ can be represented in terms of $c_{r}$ and $c_{l}$ as follows:

$$
\begin{aligned}
& \beta=\arccos \left(\frac{c_{l}\left(Z-Z_{c}\right)}{f C_{r}^{-} E_{r}}\right) \\
& \beta=\arccos \left(\frac{c_{l}\left(Z+Z_{c}\right)}{f C_{l}^{-} E_{l}}\right)
\end{aligned}
$$

Clearly, $c_{r}$ and $c_{l}$ have inverse relationship while the yaw angle $\beta$ is small enough. The length of $c_{r}^{-} n$ increases and $c_{l}^{-} n$ decreases if we increase the yaw angle $\beta$. By taking advantage of this property, the compensation formula for $y$-axis can be designed as follows:

$$
\Delta x=\left\{\begin{array}{l}
\left(1-\frac{c_{r}}{c_{l}}\right)\left(c_{l}-e_{l}\right), \text { if } \beta<0 \\
\left(1-\frac{c_{l}}{c_{r}}\right)\left(c_{r}-e_{r}\right), \text { if } \beta \geq 0
\end{array}\right.
$$




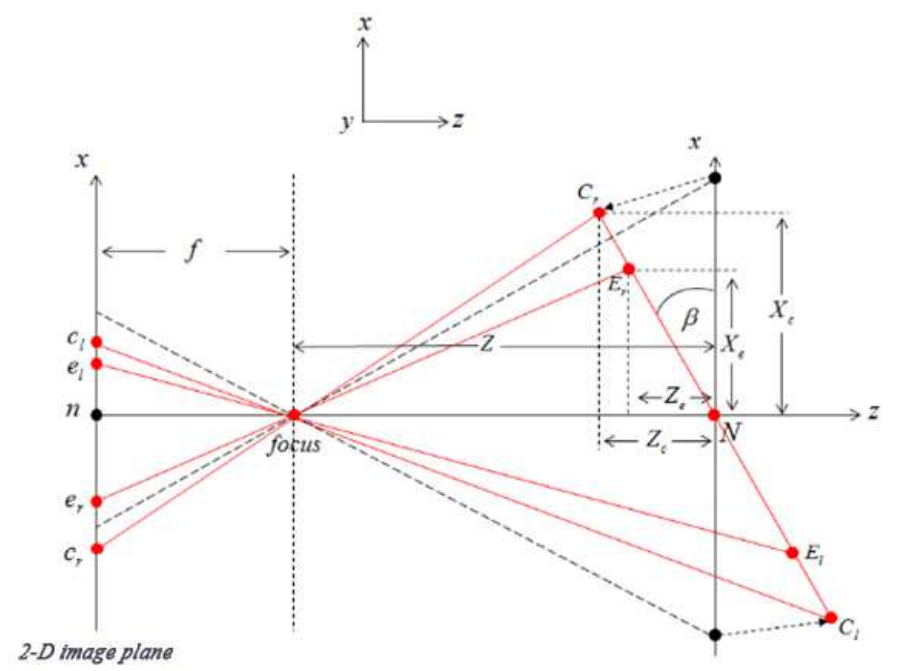

Fig. 4. Geometric perspective of the yaw rotation.

$$
\text { Center }_{\text {yaw }}\left(x^{\prime}, y\right)=\text { Center }_{\text {origin }}(x+\Delta x, y)
$$

where Center $_{\text {origin }}$ is the original location of the central point of face and Center $_{y a w}$ is the compensated location of the central point of face.

Compensation of roll: Outer corner of eyes are very robust facial feature points for adjusting roll rotation. Therefore, we only select the outer corner of eyes as the facial feature points in the compensation task, as shown in Fig. 2. For roll rotation, the coordinates of $\mathrm{Er}$ and $\mathrm{El}$ are only change in $\mathrm{x}$ and $\mathrm{y}$ axis, respectively, and the distance between the camera focus and the central point of Er and El are fixed. It means that the roll angle has same value with the rotation angle of human face displayed in Fig. 5. From Fig. 5(b), the roll angle $\alpha$ can be written as follows:

$$
\alpha=\arctan \left(\frac{Y_{e}}{X_{e}}\right)
$$

where $X_{e}=e_{r}^{x}-e_{l}^{x}$ and $Y_{e}=e_{r}^{y}-e_{l}^{y}$. In order to adjust the face to the frontal direction, we directly rotate the face image by performing the affine transform as follows:

$$
M=\left[\begin{array}{cc}
\cos \alpha & -\sin \alpha \\
\sin \alpha & \cos \alpha
\end{array}\right]
$$




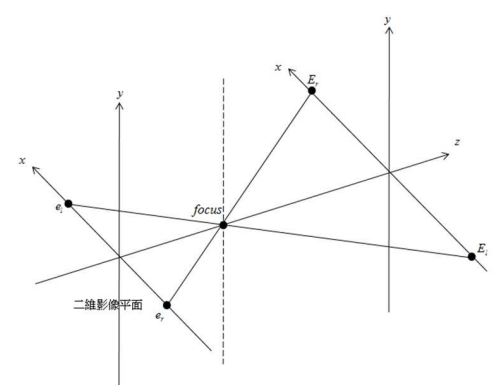

(a)

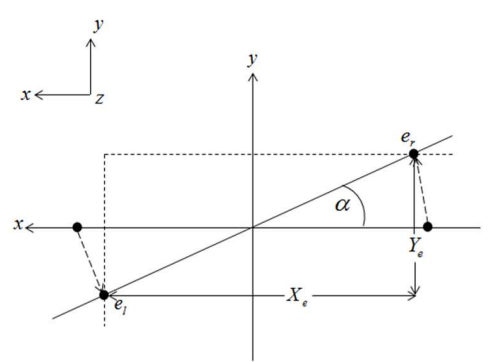

(b)

Fig. 5. Geometric perspective of the roll rotation in (a) 3-D space (b) 2-D space.

\section{Experiment Results}

This section demonstrates alignment method, which can significantly enhance the performance of facial recognition system. The FEI database [?] that includes 14 images for each of 200 individuals are selected to evaluate the effect of face alignment. For each individual, only 9 images without extreme illumination and yaw rotation are considered in the evaluation process. Fig. 6 describes the classification result of SRC [21] with our proposed face alignment processes. It outperforms SRC without alignment by $10 \%$ under all input dimension. That is, face alignment plays a vital roles to the classification work of SRC.

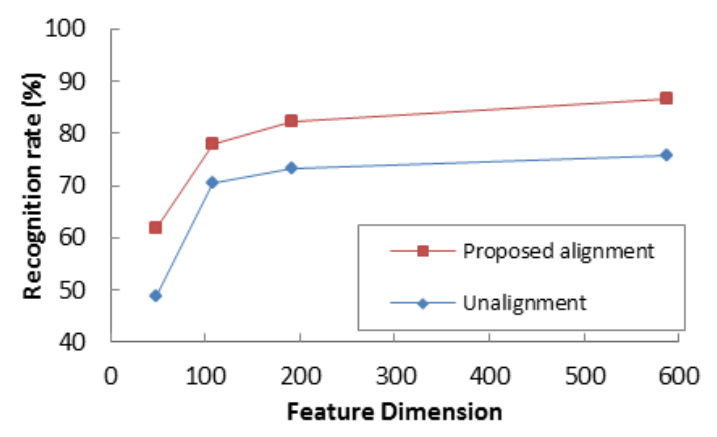

Fig. 6. FEI database recognition result of SRC under various input.

\section{Conclusions}

This paper proposes a facial recognition system with sparse representation classifier, which has been proven to be an effective classifier for illumination, occlusion, 
and corruption problems. The proposed system significantly improves the performance of facial recognition system with the help of face alignment method.In the proposed system, the pose-correction process is only applies to the $z$-axis. Further, the proposed system will apply to address the pose-correction to $x$-axis and $y$-axis, because 3D human face model helps to understand the geometric relationship between different human poses and based on this knowledge, every poor-aligned face image can be correct to frontal pose.

\section{References}

1. Viola, P. and Jones, M.J.: Robust Real-time Face Detection. International Journal of Computer Vision, 57, 2, 137-154 (2004)

2. Niyogi, S. and Freeman, W.T.: Example-based head tracking. In: 2nd IEEE International Conference on Automatic Face and Gesture Recognition, pp. 374-378. IEEE (1996)

3. De Vel, O. and Aeberhard, S.: Line-based face recognition under varying pose. IEEE Transsactions on Pattern Analysis and Machine Intelligence, 21, 10, 1081$1088(1999)$

4. Sherrah, J., Gong, S. and Ong, E.J.: Face distributions in similarity space under varying head pose. Image and Vision Computing, 19, 12, 807-819 (2001)

5. Huang, J., Shao, X. and Wechsler, H.: Face Pose Discrimination Using Support Vector Machines (SVM). In: 14th International Conference on Pattern Recognition, 1, pp. 154-156 (1998)

6. Jones, M. and Viola, P.: Fast Multi-view Face Detection. Mitsubishi Electric Research Lab TR2000396, 3, 14 (2003)

7. Rowley, H.A., Baluja, S. and Kanade, T.: Rotation invariant neural network-based face detection. In: IEEE Computer Society Conference on Computer Vision and Pattern Recognition, pp. 38-44. IEEE (1998)

8. Liu, Q., Deng, J. and Tao, D.: Dual Sparse Constrained Cascade Regression for Robust Face Alignment. IEEE Transactions on Image Processing, 25, 2, 700-712 (2016)

9. Li, Y., Gong, S., Sherrah, J. and Liddell, H.: Support vector machine based multiview face detection and recognition. Image and Vision Computing, 22, 5, 413427 (2004)

10. Moon, H. and Miller, M.L.: Estimating facial pose from a sparse representation. In: International Conference on Image Processing, 1, pp. 75-78 (2004)

11. Seemann, E., Nickel, K. and Stiefelhagen, R.: Head pose estimation using stereo vision for human-robot interaction. In: 6th IEEE International Conference on Automatic Face and Gesture Recognition, pp. 626-631. IEEE (2004)

12. Fu, Y. and Huang, T.S.: Graph Embedded Analysis for Head Pose Estimation. In: 7th IEEE International Conference on Automatic Face and Gesture Recognition, pp. 68. IEEE (2006)

13. Ren, S., Cao, X., Wei, Y. and Sun, J.: Face Alignment via Regressing Local Binary Features. IEEE Transactions on Image Processing, 25, 3, 1233-1245 (2016)

14. Tai, Y., Yang, J., Zhang, Y., Luo, L., Qian, J. and Chen, Y.: Face Recognition with Pose Variations and Misalignment via Orthogonal Procrustes Regression. IEEE Transactions on Image Processing, 25, 6, 2673-2683 (2016)

15. Wu, J. and Trivedi, M.M.: A two-stage head pose estimation framework and evaluation. Pattern Recognition, 41, 3, 1138-1158 (2008) 
16. Balasubramanian, V.N., Ye, J. and Panchanathan, S.: Biased manifold embedding: A framework for person-independent head pose estimation. In: IEEE Conference on Computer Vision and Pattern Recognition, pp. 1-7. IEEE (2007)

17. Asthana, A., Zafeiriou, S., Tzimiropoulos, G., Cheng, S. and Pantic, M.: From Pixels to Response Maps: Discriminative Image Filtering for Face Alignment in the Wild. IEEE Transactions on Pattern Analysis and Machine Intelligence, 35, 1312$1320(2015)$

18. Horprasert, T., Yacoob, Y. and Davis, L.S.: Computing 3-D head orientation from a monocular image sequence. In: 2nd IEEE International Conference Automatic Face and Gesture Recognition, pp. 244252. IEEE (1996)

19. Wang, J.G. and Sung, E.: EM enhancement of 3D head pose estimated by point at infinity. Image and Vision Computing, 25, 12, 1864-1874 (2007)

20. Kazemi, V. and Sullivan, J.: One Millisecond Face Alignment with an Ensemble of Regression Trees. In IEEE Conference on Computer Vision and Pattern Recognition, pp. 1867-1874. IEEE (2014)

21. Wright, J., Yang, A.Y., Ganesh, A., Sastry, S.S. and Ma, Y.: Robust face recognition via sparse representation. IEEE Transactions on Pattern Analysis and Machine Intelligence, 31, 2, 210-227 (2009) 